\title{
Tetrazine-trans-Cyclooctene Chemistry Applied to Fabricate Self- Assembled Fluorescent and Radioactive Nanoparticles for in Vivo Dual Mode Imaging
}

\author{
Arthur H. A. M. van Onzen, ${ }^{\dagger}$ Raffaella Rossin, ${ }^{\ddagger}$ Albertus P. H. J. Schenning, ${ }^{\S}$ Klaas Nicolay, ${ }^{\#, \|}$
}

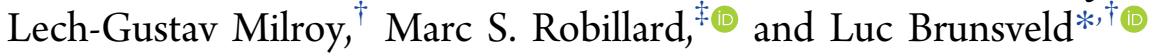

${ }^{\dagger}$ Laboratory of Chemical Biology, Department of Biomedical Engineering and Institute for Complex Molecular Systems (ICMS), Eindhoven University of Technology, P.O. Box 513, 5600 MB Eindhoven, The Netherlands

${ }^{\ddagger}$ Tagworks Pharmaceuticals, c/o Radboud University Medical Center, Department of Nuclear Medicine and Radiology, P.O. Box 9101, 6500 HB Nijmegen, The Netherlands

${ }^{\S}$ Stimuli-responsive Functional Materials and Devices and Institute for Complex Molecular Systems, Eindhoven University of Technology, P.O. Box 513, 5600 MB Eindhoven, The Netherlands

"Biomedical NMR, Department of Biomedical Engineering, Eindhoven University of Technology, P.O. Box 513, 5600 MB

Eindhoven, The Netherlands

Supporting Information

ABSTRACT: Multimodal imaging agents combine two or more imaging modalities into one probe. Self-assembling fluorescent nanoparticles are a promising class of modular multimodal imaging probes as they can allow easy blending of imaging and targeting modalities. Our group recently developed a class of self-assembling and intrinsically fluorescent small molecule-based nanoparticles (SMNPs) with excellent optical properties. In this article, we describe the efficient radiolabeling of these SMNPs via a two-step bioconjugation strategy involving the inverse-electron-demand Diels-Alder ligation between a tetrazine $(\mathrm{Tz})$-tagged radiolabel and a transcyclooctene (TCO)-tagged fluorescent small molecule building block of the SMNPs. Studies in mice revealed that the SMNPs

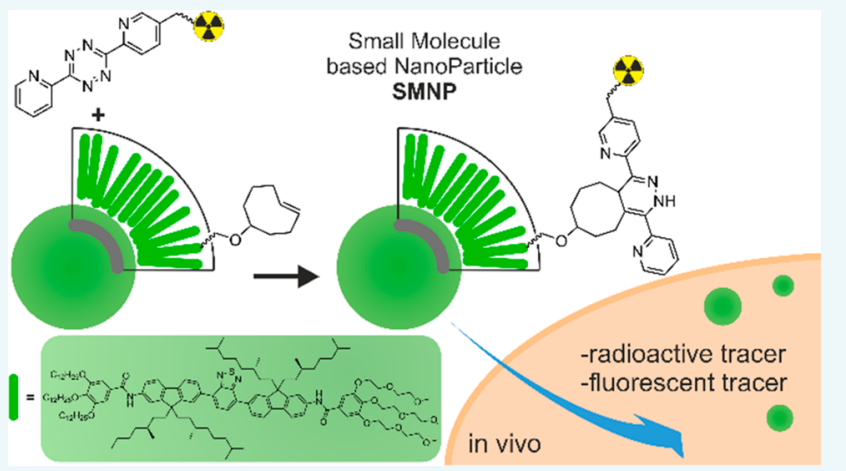
are well tolerated and could be monitored by both radioactivity and fluorescence, thereby demonstrating the potential of SMNPs in optical and dual-mode imaging in vivo. The work also testifies to the utility of the Tz-TCO conjugation chemistry for the labeling of self-assembled nanoparticles.

$\mathrm{M}$ ultimodal imaging merges two or more imaging modalities in one probe, combining the advantages of the individual techniques. ${ }^{1}$ For instance, significant research has been conducted to develop probes for dual magnetic resonance imaging (MRI), which features no tissue penetrating limit, and real-time optical imaging., ${ }^{2,3}$ Nanoparticles are a promising class of multimodal imaging agents as the blending of their specific imaging components typically does not affect the pharmacokinetic profile of the nanoparticle, frequently a disadvantage for small molecular probes. ${ }^{4}$ Furthermore, nanoparticles can be decorated with a variety of targeting moieties, giving rise to useful multivalent modes of interaction and functionality. ${ }^{5}$ Nanoparticles self-assembled from small molecular building blocks provide facile points of entries into tuning of materials properties and have shown potential for bioimaging. ${ }^{6-9}$ We have previously reported on a class of $\pi$ conjugated small molecule-based nanoparticles (SMNP) with high fluorescent quantum yields. ${ }^{10-12}$ The small molecular building blocks consist of short $\pi$-conjugated oligomers that can be functionalized with side-chains of different polarity and with bioactive functionalities at the extremities of inert ethylene glycol side chains. Tuning of the side-chain properties allows for control over molecular polarity, generation of amphiphilic analogues, and particle assembly characteristics. $^{11,12}$ The modular nature of these building blocks and their self-assembly characteristics also allows the blending of differently functionalized amphiphilic small-molecules (amps, Scheme 1) to produce spherical, amorphous SMNPs with different, useful properties for in vitro applications. ${ }^{12,13}$ Despite this potential, though, the use of such SMNPs as optical, multimodal imaging agents has not been investigated in vivo. In this regard, the introduction of a radiolabel would be beneficial as it would act as a sensitive, orthogonal readout on the

Received: January 16, 2019

Revised: February 6, 2019

Published: February 7, 2019 
Scheme 1. (A) Chemical Structures of amp-inert ${ }^{11}$ and amp-TCO, $\pi$-Conjugated Small Molecules, Which Self Assemble into SMNPs. (B) Two-Step Radiolabeling Strategy Starts with Chelation of the Radioisotope to the Tetrazine-DOTA Derivative Followed by (C) Conjugation to the SMNP Co-Self-Assembled from amp-inert and amp-TCO
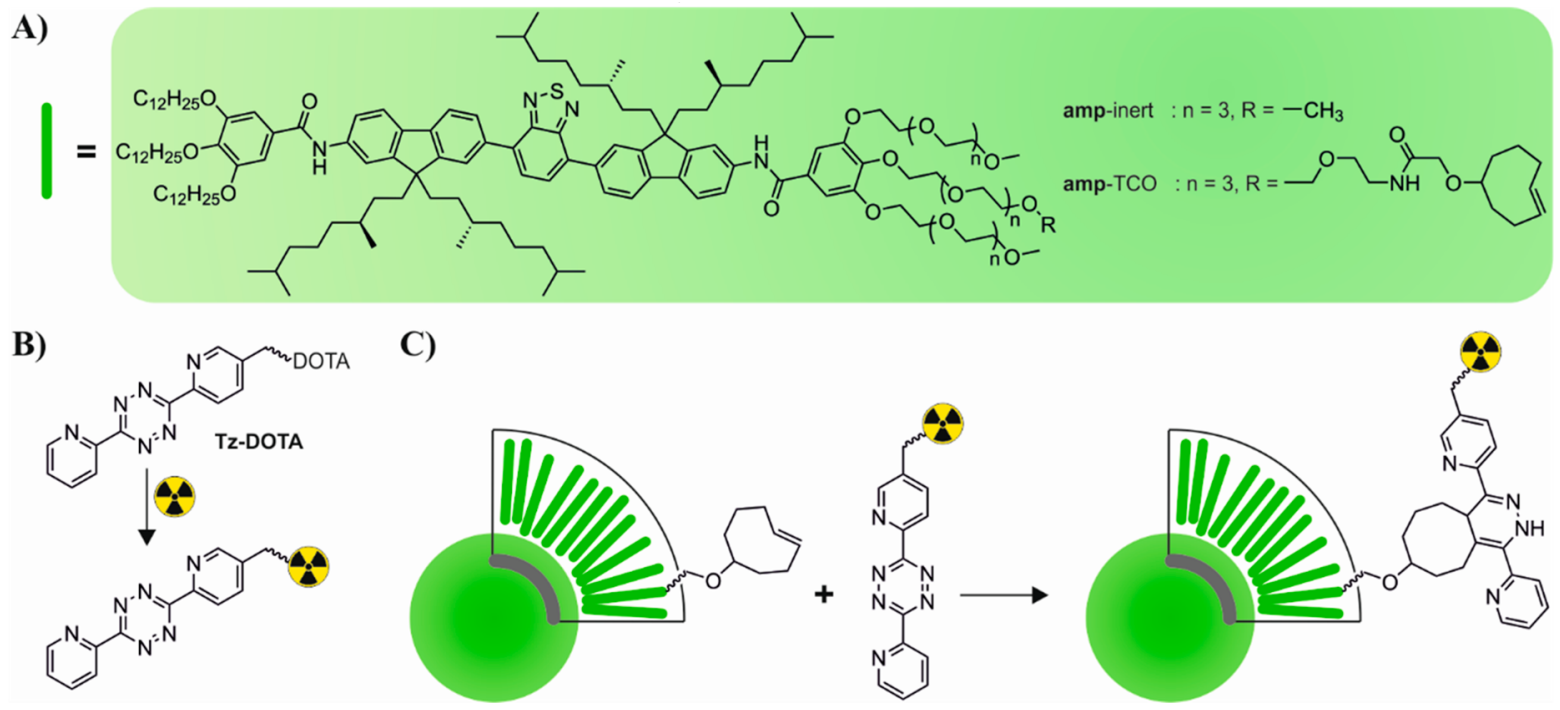

biodistribution of the SMNPs in tissues and organs. Here, we report the efficient conjugation of a radiolabel to a SMNP making use of the bio-orthogonal inverse-electron-demand Diels-Alder (IEDDA) reaction between a tetrazine $(\mathrm{Tz})$ and trans-cyclooctene (TCO) $)^{14-16}$ (Scheme 1) and subsequently assess the potential of the resulting conjugated SMNPs as in vivo dual-mode imaging agents.

Our radiolabeling strategy centered on the efficient conjugation of a Tz-DOTA derivative, prechelated to the radioisotope, to preassembled SMNPs. This maximized flexibility, while minimizing the number of radioactive handling steps. Radiolabeling of the Tz-DOTA was performed as described in the literature. ${ }^{17}$ Briefly, a small amount of TzDOTA was dissolved in $0.2 \mathrm{M}$ ammonium acetate buffer $(\mathrm{pH}$ 5.5) followed by the addition of indium-111. After $5 \mathrm{~min}$ incubation at $60{ }^{\circ} \mathrm{C}$ the solution was treated with DTPA to yield $>99.7 \%{ }^{111}$ In-labeled Tz-DOTA (based on iTLC). A small molecule building block featuring a reactive transcyclooctene unit (amp-TCO, Scheme 1) was synthesized and coassembled with amp-inert (25\% amp-TCO) into SMNPs in a facile manner. Next, the Tz-DOTA derivative was reacted with the TCO-bearing SMNPs in buffer over $30 \mathrm{~min}$. Reversed-phase (RP)-TLC studies on the ${ }^{111}$ In-labeled TzDOTA and SMNPs (Figure 1A) revealed the extent of the ${ }^{111}$ In-labeling. Whereas the unreacted ${ }^{111}$ In-labeled Tz-DOTA eluted with an $R_{\mathrm{f}}=0.3$, with and without amp-inert as a control, most of the indium-111 activity was observed to colocalize with the SMNPs at the baseline $\left(R_{\mathrm{f}}<0.1\right)$ after conjugation. The low activity observed at $R_{\mathrm{f}}>0.1$, corresponding to residual unreacted Tz-DOTA or free indium-111, could be removed by size exclusion chromatography. Analysis of the reaction mixtures using a phosphorimager (Figure 1B) or under $365 \mathrm{~nm}$ UV-light (Figure 1C) also indicated almost full conversion of the Tz-DOTA based on the colocalization of the indium-111 radio activity and the SMNP fluorescence signal. Besides the successful Tz-DOTA conjugation using the amp-TCO building block, these results indicate that all radioisotope labeling is specifically incorporated through a covalent reaction between the ${ }^{111} \mathrm{In}$-labeled TzDOTA and the amp-TCO, and not due to nonspecific,

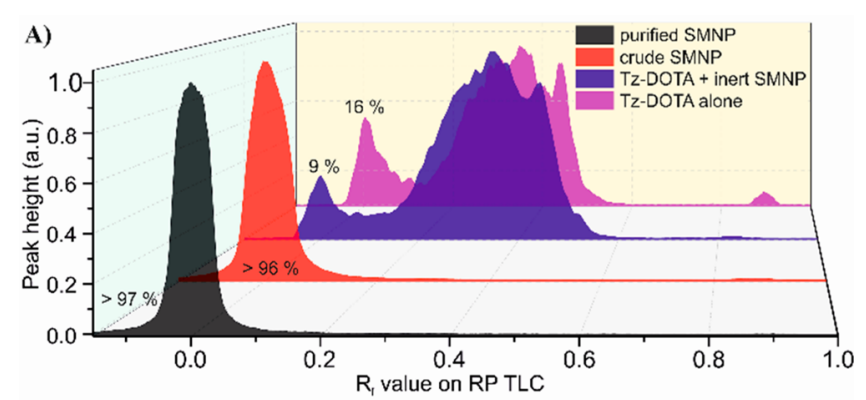

В)

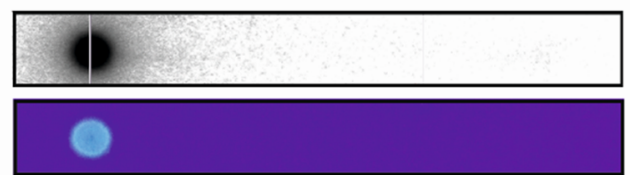

Figure 1. (A) Reversed phase (RP)-TLC using 50\% acetonitrile/ water of ${ }^{111}$ In-labeled Tz-DOTA (magenta), ${ }^{111}$ In-labeled Tz-DOTA with inert-SMNPs (blue), ${ }^{111}$ In-labeled Tz-DOTA combined with SMNPs featuring with $25 \%$ amp-TCO before (red) and after purification by size exclusion chromatography (black). (B) RP-TLC of purified ${ }^{111}$ In-labeled SMNP imaged with phosphorimager and (C) fluorescence.

noncovalent incorporation of the Tz-DOTA or indium-111 label into the SMNPs.

Two other radiolabeling strategies were also investigated for the conjugation of the indium-111 to SMNPs: one based on SMNPs self-assembled with a DOTA connected building block (amp-DOTA), the other on a two-step labeling protocol using a bicyclononyne-DOTA conjugate (BCN-DOTA) (see SI for synthesis and characterization details). SMNPs composed of amp-DOTA could be radiolabeled with indium-111 with an efficiency of $45 \%$. The excess of radioactive indium-111 could in part be removed via extensive purification by size exclusion column chromatography, leading to $88 \%$ of the radioactivity resulting from the SMNPs (SI Figure S5). While promising, this labeling efficiency was nonetheless too low for in vivo applications. The BCN-DOTA derivative by contrast did not show any reaction with the SMNPs assembled from amp-azide 
monomers, presumably due to the azide group's lack of solvent accessibility within the SMNPs. The limitations of these two strategies combined with the results obtained for the Tz-TCO conjugation strategy, testify to the suitability of the latter for use with SMNPs.

The particle and materials characteristics of the SMNP before and after the Tz-DOTA conjugation via the IEDDA reaction were further evaluated using cold-control studies. SMNPs consisting of $25 \%$ of amp-TCO and $75 \%$ amp-inert, measured an average diameter of $90 \mathrm{~nm}$ by DLS. Incubating the same SMNPs in serum did not influence their optical properties (Figure 2). The quantum yield of the SMNPs was
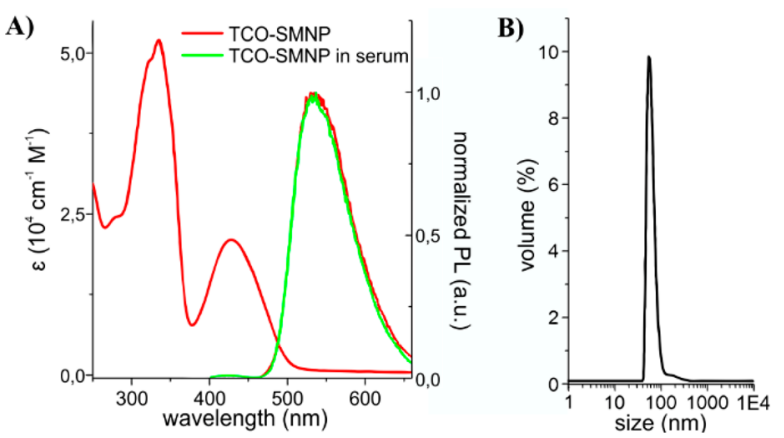

Figure 2. (A) Excitation (left) and emission spectra (right) of $25 \%$ TCO-SMNPs in water and after $17 \mathrm{~h}$ incubation in serum at $37^{\circ} \mathrm{C}$. (B) Hydrodynamic diameter of SMNPs containing $25 \% \mathrm{amp}-\mathrm{TCO}$ in water.

determined to be 0.46 and the absorption cross section was 6.7 $\times 10^{-12} \mathrm{~cm}^{2}$. Coupling of Tz-DOTA to the SMNPs did not influence the size or optical properties of the particles, nor did subsequent purification by size exclusion chromatography (SI Figure S9).

The promising optical and structural characteristics of the SMNPs and their facile radiolabeling, prompted the study of their functioning as dual-imaging agents in vivo. The ${ }^{111} \mathrm{In}$ labeled SMNPs were administrated intravenously to mice, to profile their intrinsic in vivo biodistribution and clearance. The mice did not show any changes in behavior or activity over a period of 3 days, indicating that the SMNPs were well tolerated by the mice at peak blood concentrations of $30 \mu \mathrm{M}$. Blood samples taken via the vena saphena were weighed and analyzed by $\gamma$-counter (Figure 3A insert). Heparin was present during blood sampling to increase the efficiency of sample handling during the subsequent fluorescence analysis. After injection of the SMNPs, the observed percentage dose per gram in blood was measured to be $17.3 \pm 1.3 \mathrm{ID} / \mathrm{g}$ after $2 \mathrm{~min}$, which dropped to $4.4 \pm 0.9 \% \mathrm{ID} / \mathrm{g}$ after $5 \mathrm{~min}$, followed by an average value of $0.9 \pm 0.2 \% \mathrm{ID} / \mathrm{g}$ after $10 \mathrm{~min}$, indicating that most SMNPs were efficiently cleared from the bloodstream. The biodistribution of the SMNPs was determined periodically $(4,24$, and $70 \mathrm{~h}$ post administration of SMNPs; Figure $3 \mathrm{~A})$ by harvesting the organs at the indicated times after anesthetized cervical dislocation and then weighing and $\gamma$-counting the percentage injected dose per gram tissue (\% ID/g). At the $4 \mathrm{~h}$ time point, the SMNPs had been predominantly taken up in the liver with $80.4 \pm 3.7 \% \mathrm{ID} / \mathrm{g}$, with significant uptake measured in the spleen as well $(34.8 \pm 1.8 \% \mathrm{ID} / \mathrm{g})$. These observations are possibly explained by mononuclear phagocytic system (MPS) elimination by macrophages localized in the liver (Kupffer cells) and the spleen (red pulp) as is

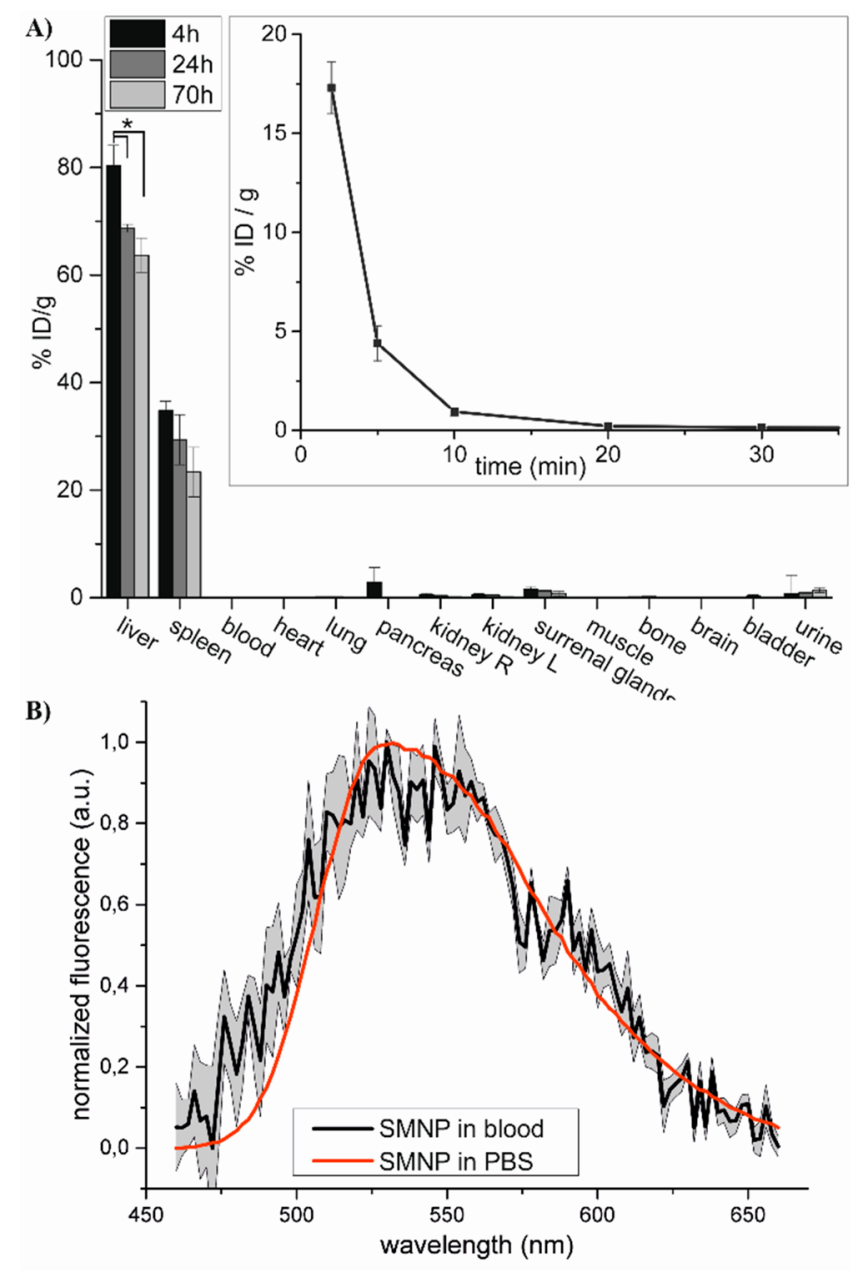

Figure 3. (A) Biodistribution of SMNPs in mice 4, 24, and $70 \mathrm{~h}$ after i.v. administration (error bar represents SEM, $n=3$, $* p<0.05$ ). (inset) blood clearance of SMNPs in mice (error bar represents SEM, $n=4)$. (B) Fluorescence spectrum of SMNPs in blood (black line), corrected for non-SMNP containing blood, and fluorescence spectrum of SMNPs in PBS (red line). Error area represents SD, $n$ $=3$.

commonly observed. ${ }^{18}$ No uptake in lung tissue was observed, which is expected for nonaggregated particles smaller than $2000 \mathrm{~nm}$ in diameter. ${ }^{19}$

The SMNPs levels in liver and spleen decreased after 24 and $70 \mathrm{~h}$, which is indicative of effective excretion from the body, possibly after SMNP disassembly, via solvation into hydrophobic compartments. ${ }^{19}$ In this respect, it is worth mentioning that Tz-DOTA itself has been reported elsewhere to clear rapidly via the kidneys. ${ }^{17,20}$ By comparison, no renal clearance was observed for the SMNPs, strengthening the conclusion that all DOTA present is covalently attached via the tetrazineTCO conjugation chemistry and not nonspecifically incorporated in the SMNP. ${ }^{21}$

Besides the radiotracer imaging, the fluorescence of the SMNPs was evaluated as a second mode of imaging. The liver tissue's autofluorescent properties prevented detection of the SMNPs by microscopy imaging in this organ. Emission spectra could, however, be recorded for the SMNPs in blood samples collected after SMNP injection. Subtraction of blood background fluorescence clearly revealed the typical fluorescence signature of the SMNPs (Figure 3B). While scattering effects 
of the blood ${ }^{22}$ probably account for the fluctuations observed for the SMNP spectrum, the spectral profile shape fits well with the one measured in PBS, indicating that these SMNPs are indeed detectable in complex blood tissue.

In summary, we have shown that intrinsically fluorescent self-assembled nanoparticles (SMNP) prepared through coassembly of $\pi$-conjugated building blocks could be efficiently radiolabeled by the IEDDA reaction between a ${ }^{111}$ In-labeled tetrazine-DOTA derivative and a TCO-SMNP. Dual radiotracer and fluorescence imaging studies revealed a rapid clearance of the SMNPs from the blood, and efficient uptake in the liver and spleen with subsequent clearance. These first studies serve to demonstrate the potential of self-assembled, small molecule based, nanoparticles as dual mode imaging agents. However, further improvements are needed. For example, fluorescence-based detection in liver tissue should be possible by tuning the absorption and emission characteristics through chemical modification of the constituent monomers. ${ }^{23}$ More extensive PEGylation of the SMNPs would potentially confer stealth-like properties for applications requiring longer circulation times. ${ }^{19}$ In this respect, the selectivity and efficiency of the IEDDA reaction should facilitate access to such modified SMNPs.

\section{ASSOCIATED CONTENT}

\section{S Supporting Information}

The Supporting Information is available free of charge on the ACS Publications website at DOI: 10.1021/acs.bioconjchem.9b00038.

Materials, methods, synthetic procedures, analytical data and control experiments (PDF)

\section{AUTHOR INFORMATION}

\section{Corresponding Author}

*E-mail: 1.brunsveld@tue.nl.

\section{ORCID $\odot$}

Albertus P. H. J. Schenning: 0000-0002-3485-1984

Marc S. Robillard: 0000-0002-3690-2087

Luc Brunsveld: 0000-0001-5675-511X

\section{Notes}

The authors declare the following competing financial interest(s): R. Rossin and M.S. Robillard declare competing financial interest as employees and shareholders of Tagworks. \#Deceased January 10, 2017

\section{ACKNOWLEDGMENTS}

This work is supported by NanoNextNL, a micro and nanotechnology consortium of the government of The Netherlands and 130 partners and The Netherlands Organisation for Scientific Research (NWO) via Gravity Program 024.001.035. Ralf Bovee and Dr. Xianwen Lou are thanked for their help with analytical measurements and Leonie Niesen for help with in vivo experiments.

\section{REFERENCES}

(1) Jennings, L. E., and Long, N. J. (2009) 'Two is better than one'-probes for dual-modality molecular imaging. Chem. Commun., 3511-3524.

(2) Louie, A. (2010) Multimodality Imaging Probes: Design and Challenges. Chem. Rev. 110, 3146-3195.

(3) van Dam, G. M., Themelis, G., Crane, L. M. A., Harlaar, N. J., Pleijhuis, R. G., Kelder, W., Sarantopoulos, A., de Jong, J. S., Arts, H. J.
G., van der Zee, A. G. J., Bart, J., Low, P. S., and Ntziachristos, V. (2011) Intraoperative tumor-specific fluorescence imaging in ovarian cancer by folate receptor- $\alpha$ targeting: first in-human results. Nat. Med. $17,1315-1319$.

(4) Leary, J., and Key, J. (2014) Nanoparticles for multimodal in vivo imaging in nanomedicine. Int. J. Nanomed. 9, 711-726.

(5) Lee, D.-E., Koo, H., Sun, I.-C., Ryu, J. H., Kim, K., and Kwon, I. C. (2012) Multifunctional nanoparticles for multimodal imaging and theragnosis. Chem. Soc. Rev. 41, 2656-2672.

(6) Schill, J., Schenning, A. P. H. J., and Brunsveld, L. (2015) SelfAssembled Fluorescent Nanoparticles from $\pi$-Conjugated Small Molecules: En Route to Biological Applications. Macromol. Rapid Commun. 36, 1306-1321.

(7) Hao, Y., Zheng, C., Wang, L., Hu, Y., Guo, H., Song, Q., Zhang, H., Zhang, Z., and Zhang, Y. (2017) Covalent self-assembled nanoparticles with $\mathrm{pH}$-dependent enhanced tumor retention and drug release for improving tumor therapeutic efficiency. J. Mater. Chem. B 5, 2133-2144.

(8) Xu, X., Liu, R., and Li, L. (2015) Nanoparticles made of $\pi$ conjugated compounds targeted for chemical and biological applications. Chem. Commun. 51, 16733-16749.

(9) Ardizzone, A., Kurhuzenkau, S., Illa-Tuset, S., Faraudo, J., Bondar, M., Hagan, D., Van Stryland, E. W., Painelli, A., Sissa, C., Feiner, N., Albertazzi, L., Veciana, J., and Ventosa, N. (2018) Nanostructuring Lipophilic Dyes in Water Using Stable Vesicles, Quatsomes, as Scaffolds and Their Use as Probes for Bioimaging. Small 14, 1703851.

(10) Kaeser, A., Fischer, I., Abbel, R., Besenius, P., Dasgupta, D., Gillisen, M. A. J., Portale, G., Stevens, A. L., Herz, L. M., and Schenning, A. P. H. J. (2013) Side Chains Control Dynamics and Self-Sorting in Fluorescent Organic Nanoparticles. ACS Nano 7, 408416.

(11) Petkau, K., Kaeser, A., Fischer, I., Brunsveld, L., and Schenning, A. P. H. J. (2011) Pre- and Postfunctionalized Self-Assembled $\pi$ Conjugated Fluorescent Organic Nanoparticles for Dual Targeting. J. Am. Chem. Soc. 133, 17063-17071.

(12) Fischer, I., Petkau-Milroy, K., Dorland, Y. L., Schenning, A. P. H. J., and Brunsveld, L. (2013) Self-Assembled Fluorescent Organic Nanoparticles for Live-Cell Imaging. Chem. - Eur. J. 19, 16646-16650.

(13) van Onzen, A. H. A. M., Albertazzi, L., Schenning, A. P. H. J., Milroy, L.-G., and Brunsveld, L. (2017) Hydrophobicity determines the fate of self-assembled fluorescent nanoparticles in cells. Chem. Commun. 53, 1626-1629.

(14) Meyer, J.-P., Adumeau, P., Lewis, J. S., and Zeglis, B. M. (2016) Click Chemistry and Radiochemistry: The First 10 Years. Bioconjugate Chem. 27, 2791-2807.

(15) Knall, A.-C., and Slugovc, C. (2013) Inverse electron demand Diels-Alder (iEDDA)-initiated conjugation: a (high) potential click chemistry scheme. Chem. Soc. Rev. 42, 5131-5142.

(16) Oliveira, B. L., Guo, Z., and Bernardes, G. J. L. (2017) Inverse electron demand Diels-Alder reactions in chemical biology. Chem. Soc. Rev. 46, 4895-4950.

(17) Rossin, R., Renart Verkerk, P., van den Bosch, S. M., Vulders, R. C. M., Verel, I., Lub, J., and Robillard, M. S. (2010) In Vivo Chemistry for Pretargeted Tumor Imaging in Live Mice. Angew. Chem., Int. Ed. 49, 3375-3378.

(18) Li, S.-D., and Huang, L. (2008) Pharmacokinetics and Biodistribution of Nanoparticles. Mol. Pharmaceutics 5, 496-504.

(19) Blanco, E., Shen, H., and Ferrari, M. (2015) Principles of nanoparticle design for overcoming biological barriers to drug delivery. Nat. Biotechnol. 33, 941-951.

(20) Rossin, R., Läppchen, T., van den Bosch, S. M., Laforest, R., and Robillard, M. S. (2013) Diels-Alder Reaction for Tumor Pretargeting: In Vivo Chemistry Can Boost Tumor Radiation Dose Compared with Directly Labeled Antibody. J. Nucl. Med. 54, 19891995.

(21) Longmire, M., Choyke, P. L., and Kobayashi, H. (2008) Clearance properties of nano-sized particles and molecules as imaging agents: considerations and caveats. Nanomedicine 3, 703-717. 
(22) Bosschaart, N., Edelman, G. J., Aalders, M. C. G., van Leeuwen, T. G., and Faber, D. J. (2014) A literature review and novel theoretical approach on the optical properties of whole blood. Lasers Med. Sci. 29, 453-479.

(23) Pennakalathil, J., Jahja, E., Özdemir, E. S., Konu, Ö., and Tuncel, D. (2014) Red Emitting, Cucurbituril-Capped, pHResponsive Conjugated Oligomer-Based Nanoparticles for Drug Delivery and Cellular Imaging. Biomacromolecules 15, 3366-3374. 